\title{
Valoración de la uretrorragia en el niño
}

\author{
Domínguez Hinarejos C, Bonillo García MA, Alapont Alacreu JM, Serrano Durbá A, \\ Estornell Moragues F, García Ibarra F.
}

Servicio de Urología Infantil. Hospital Universitario La Fe. Valencia.

Actas Urol Esp. 2007;31(1):29-32

\begin{abstract}
RESUMEN
VALORACIÓN DE LA URETRORRAGIA EN EL NIÑO

Objetivo: Revisar los casos de uretrorragia, la necesidad de exploraciones complementarias, su manejo e historia natural.

Material y Métodos: Analizamos retrospectivamente 12 pacientes que presentaron una uretrorragia, diagnosticados de forma consecutiva en nuestro centro. Se evaluaron parámetros como la edad, tiempo de evolución y hábito miccional. De igual modo, describimos las técnicas diagnósticas utilizadas, el manejo terapéutico y la evolución del paciente.

Resultados: Todos los casos corresponden a varones con edad media de 8,7 años (18 meses y 14 años). Los episodios de uretrorragia aparecen en el $100 \%$ de los pacientes, mientras que fueron diarios en el 58,4\%. El 33\% (4/12) se acompañan además de otra sintomatología miccional. El 75\% (9/12) presentaban un hábito miccional perezoso. Entre los antecedentes urológicos destacan: 1 paciente circuncidado hacía 3 años, otro al que se le practicó una meatotomía por estenosis del meato 2 años antes y por último, uno al que se le realizó una electrocoagulación de un pólipo uretral hacía un mes. El 83,4\% (10/12) de los urinocultivos realizados fueron negativos. Se practicó uretrocistoscopia en 9 pacientes, detectando en 4 una hipertrofia del verum, en 3 cambios inflamatorios adyacentes a la uretra membranosa, en 1 paciente esfacelos en uretra bulbar y en el último no se objetivaron hallazgos patológicos. De los 8 pacientes que presentaban un hábito miccional perezoso, el 75\% (6/8) se resolvieron espontáneamente después de corregir el hábito miccional tras una media de 9 meses. Sólo 2 precisaron tratamiento quirúrgico mediante resección endoscópica transuretral. En los otros 2 pacientes la sintomatología desapareció espontáneamente en el transcurso del seguimiento de los mismos.

Conclusiones: La uretrorragia en el niño es un proceso benigno en la gran mayoría de los casos, que se resuelve de forma espontánea prácticamente siempre durante los 2 primeros años de seguimiento. Los estudios radiológicos y las exploraciones endoscópicas resultan innecesarios en el manejo inicial de estos pacientes quedando relegadas a cuadros persistentes en el tiempo.
\end{abstract}

Palabras clave: Uretrorragia. Verum. Infancia.

\section{ABSTRACT}

ASSESMMENT URETHRORRHAGIA IN CHILDHOOD

Objetive. To review urethrorrhagia cases, the need of complementary test, its management and natural history.

Material and method. A retrospective analysis was carried out for the 12 patients who suffered from urethrorrhagia, consecutively diagnosed in our department. Parameters as age, and micturitional habit were evaluated. In this way, we described the diagnostic techniques used, assessment and the follow-up of each patient.

Results. All cases correspond to male children with an average age of 8.7 years (range: 18 months- 14 years). Urethrorrhagia appears in $100 \%$ of the patients, episodes which were daily in $58.4 \% .33 \%(4 / 12)$ of them also present other micturitional symptoms. $75 \%(9 / 12)$ had a lazy micturitional habit. Among the urological backgrounds we found: 1 patient was circumcised 3 years ago, a meatotomy was done in other due to meatus stenosis 2 years before, and a last one had an electrocoagulation of a urethral polyp just 1 month before. 83.4\% (10/12) of urine cultures were negative. An urethrocystoscope was done in 9 patients finding a verum hypertrophy in 4, inflammatory changes next to membranous urethra in 3, scar at the bulbar urethra in 1 and in a last one no abnormalities were found. Out of 8 patients with lazy micturitional habit, $75 \%(6 / 8)$ resolve spontaneously once they correct their habits after a mean follow-up of 9 months. Only 2 patients needed surgery (TUR). In the other 2 patient, symptoms.

Conclusions. Urethrorrhagia in childhood is a benign condition in most cases, which is almost always cured spontaneously during the first 2 years of follow-up. Radiological studies as well as endoscopic procedures are unnecessary in the early management of these patients thus being relegated to recurrent or persistent bleeding.

Keywords: Urethrorrhagia. Verum. Childhood. 
Desde que en 1982 Kaplan $^{1}$ introdujera el término de uretrorragia idiopática para referirse al marcado hemático en la ropa interior del niño entre las micciones, han surgido en la literatura médica diferentes términos, como uretritis bulbar o anterior del adolescente ${ }^{2-4}$, para denominar una circunstancia considerada a veces anodina pero que en realidad su historia natural no es del todo conocida. Si bien existe una diferencia conceptual entre uretrorragia y hematuria, desde el punto de vista clínico es muy difícil diferenciarlas en la mayoría de los casos, sobre todo en niños. Por otro lado, es un tema polémico por la angustia y preocupación, en cierto modo desmedida, que genera a los padres.

Clásicamente se ha aceptado su benignidad, un mero hallazgo sin relevancia clínica que sin embargo, dado su relación con gran número de patologías se presupone el diagnóstico diferencial con éstas. Entre las patologías a tener en cuenta destacamos las de etiología infecciosa ${ }^{3-5}$, la estenosis de uretra $^{6}$ y las tumoraciones uretrales ${ }^{7-9}$.

Nuestro objetivo es la revisión de los casos diagnosticados en nuestro hospital y determinar la necesidad de realizar exploraciones complementarias, justificando cuáles son necesarias y, lo más importante, cuáles podemos omitir ya que no es despreciable la yatrogenia que provocamos con exploraciones agresivas en este grupo de edad.

\section{MATERIAL Y MÉTODO}

Analizamos retrospectivamente 12 pacientes valorados en nuestro centro por presentar uretrorragia, cuya edad media fue de 8,7 años con un rango de 18 meses a 14 años.

Tras realizar una historia clínica detallada, insistiendo tanto en el tipo de manchado como en el hábito miccional, solicitamos como primer escalón diagnóstico un sedimento y cultivo de orina junto a una ecografia reno-vesical. Si no obtuvimos unos resultados que nos permitiesen filiar la etiología del sangrado, realizamos bien una cistouretrografía miccional seriada (CUMS) o una urografia intravenosa (UIV), dejando la uretrocistoscopia para la confirmación de la sospecha diagnóstica. Sólo en aquellos casos anecdóticos en los que no llegamos a un diagnóstico definitivo, solicitamos un estudio urodinámico o biopsia endoscópica. Los pacientes se sometieron a un seguimiento clínico medio de 24 meses, siendo el tiempo de citación muy variable dependiendo de la intensidad y número de episodios. No obstante, siguiendo nuestro protocolo se les controlaba semestralmente durante el primer año y después anualmente.

\section{RESULTADOS}

De los 12 pacientes con sangrado 7 fueron episodios diarios $(58,4 \%)$. La mediana del tiempo desde la presentación de la uretrorragia hasta que los pacientes acudieron a la consulta fue de 4 meses con un rango de 3 días a 2 años. Sólo dos casos tenían antecedentes urológicos de interés, un paciente había sido circuncidado 3 años antes y a otro se le practicó una meatotomía hacía 2 años por una estenosis uretral secundaria a esclerosis balanoprepucial. En cuanto al hábito miccional, 9 pacientes (75\%) presentaban uno perezoso, definido como el retraso del inicio de la micción tras el deseo miccional, 2 (16,7\%) referían un discreto síndrome miccional irritativo, y otros 2 dolor al inicio de la micción.

El cultivo de orina fue negativo en 10 pacientes $(83,4 \%)$. La ecografía y la urografía no aportaron información adicional en ninguno de los casos realizados (3 casos). En 7 pacientes solicitamos una CUMS, donde se objetivó en 2 un defecto de repleción en la fase miccional a nivel de la uretra posterior. Uno de estos hallazgos se consideró compatible con una hipertrofia del verum, mientras que el otro se interpretó como sugestivo de un pólipo uretral por lo que se realizó una uretrocistoscopia, donde sólo se objetivó cambios inflamatorios inespecíficos a nivel de la uretra membranosa. En otros 6 pacientes tamnbién se realizó uretrocistoscopia, hallándose hipertrofia del verum en 4 casos junto a distintas áreas inflamatorias, fundamentalmente a nivel de la uretra bulbar, en los otros dos fue normal. En un paciente con persistencia de la sintomatología más allá de los 24 meses se realizó estudio urodinámico que fue normal y una biopsia uretral posterior cuyo resultado se nos informó de una uretritis crónica con leve displasia uretral.

El planteamiento terapéutico inicial en todos los pacientes con hábito miccional perezoso fue la corrección del mismo, micción ante el primer 
deseo o micción cada 2-3 horas en aquellos pacientes que con el tiempo no lo presentaban por inhibición voluntaria y de esta forma conseguir la reeducación miccional. Con esta medida desapareció el sangrado uretral durante los primeros 9 meses de seguimiento en el 77,8\% (7 niños) de aquellos que se les habían etiquetado de micción perezosa, y el $100 \%$ a los 24 meses de seguimiento.

De los 2 casos donde hubo un crecimiento significativo de $E$. Coli, en uno de ellos asintomático persistiendo la uretrorragia a pesar de la administración de antibiótico ajustado al antibiograma. El otro que refería disuria se le pauto tratamiento antibiótico, con cultivo negativo finalizado el mismo, pero el sangrado persistió desapareciendo a los 15 días de tras el tratamiento.

Sólo 2 niños precisaron exploración endoscópica. Uno de ellos se sometió a una resección transuretral (RTU) por la presencia de un pólipo uretral y a nueva resección al mes después por reaparición del sangrado y presencia de esfacelos en uretra bulbar. En un segundo caso, tras una respuesta inicial a la administración empírica de antibiótico, desarrolló un curso tórpido y recurrente por lo que se electrocoaguló endoscópicamente una zona hiperémica que sangraba con facilidad a nivel de la uretra membranosa.

La duración de la uretrorragia tras el tratamiento fue igual o menor a 6 meses en 7 pacientes $(58,4 \%)$, desapareciendo en un total de 10 niños $(84,4 \%)$ durante el primer año. En aquellos 2 casos donde el proceso fue recurrente prolongándose más allá de los 24 meses, 36 en uno de ellos al que se realizó electrocoagulación por pólipo uretral y en otro desapareció espontáneamente a los 5 años de seguimiento. Ningún paciente desarrolló una estenosis uretral durante el seguimiento.

\section{DISCUSIÓN}

La gran mayoría de los urólogos pediátricos están familiarizados con niños que acuden traídos por padres angustiados por presentar un marcado hemático en su ropa interior. Si bien tradicionalmente se ha postulado su benignidad, la falta de consenso en la literatura puede dar lugar a exploraciones innecesarias y frustrados planteamientos terapéuticos.
Se han atribuido muchas causas como responsable de esta sintomatología, por ejemplo, clásicamente se relacionó la presencia de uretritis en la infancia con la estenosis de meato uretral por lo que se propuso como tratamiento la meatotomía ${ }^{10}$, pero se han notificado un importante número de niños con sangrado recurrente tras la misma como ocurrió en uno de nuestros casos. De la misma forma, durante un tiempo se justificó el tratamiento antibiótico empírico al postular la implicación de agentes infecciosos como la etiología más probable de la uretritis en niños. Sin embargo, su uso no demostró una disminución ni en la duración de los síntomas ni en la aparición de episodios recurrentes ${ }^{2}$. Al igual que otras series publicadas ${ }^{11}$, la gran mayoría de nuestros pacientes $(83,4 \%)$ presentaron un urinocultivo negativo, en sólo 2 pacientes fue positivo, pero en ambos la uretrorragia persistió a pesar de negativizarse el cultivo. La asociación de uretritis con alteraciones inmunológicas, como el síndrome de Reiter ${ }^{12}$ y ciertos estados de hipogammaglobulinemia ${ }^{12}$, han sugerido el papel de procesos autoinmunes. Si bien en ninguno de nuestros pacientes se realizaron estudios inmunohistoquímicos, la ausencia de otras manifestaciones clínicas típicas, como artritis o conjuntivitis, motivó que no se tuviera en cuenta este origen. Docimo et al. ${ }^{4}$ proponen que existe una metaplasia escamosa en la uretra anterior desencadenada por una irritación crónica cuya causa predisponente se desconoce. Este epitelio uretral frágil se alteraría con facilidad con el consiguiente sangrado y la predisposición a la estenosis uretral. Sin embargo, todas estas teorías están basadas por estudios con escasos pacientes y datos insuficientes, por lo que pensamos que excepto en los casos donde no se objetive una estenosis de uretra, se debe aceptar la benignidad de esta patología, dado que tiende a desaparecer espontáneamente.

Pensamos al igual que Herz et al. ${ }^{13}$ que este sangrado uretral es una manifestación más de un síndrome de disfunción de vaciado subyacente, que justificaría esta irritación crónica y la subsiguiente uretrorragia, motivada por el traumatismo sobre el verum durante la micción, como ocurrió en el $67 \%$ de nuestros casos, por ello en aquellos que la historia clínica no sea 
aclaratoria podemos realizar una flujometría, donde observaremos un flujo miccional elevado $u$ otros parámetros.

En cuanto a qué exploraciones complementarias realizar a estos niños, nosotros creemos que hay que ser lo menos agresivo posible y solicitar aquellas que nos sean necesarias, que supongan un menor riesgo para el niño, sin olvidar de cuales podemos prescindir. Cuando revisamos otras publicaciones, la realización de pruebas radiológicas (CUMS, ecografia, UIV) no ha aclarado el origen de este sangrado en la mayoría de los casos $^{11}$. En nuestra serie, se nos informó de 2 defectos de repleción, que no aportaron datos de interés en el posterior manejo del paciente. El papel de la uretrocistoscopia es controvertido ya que a pesar los cambios inflamatorios inespecíficos encontrados a nivel uretral, tanto en nuestro casos como en la revisión de la literatura no cambiarian la actitud terapéutica ya que en la actualidad todavía se desconoce la etiología exacta y el tratamiento efectivo para la misma. Por lo tanto, pensamos que podemos omitirla dado que es una exploración cruenta, no exenta de riesgos y además para su realización en niños es necesaria una anestesia general. Si bien Kaplan y Brock ${ }^{1,6}$ publicaran en su serie una incidencia del $20 \%$ de estenosis uretrales entre aquellos que se sometieron a una cistoscopia, otros autores ${ }^{3,4,14}$ determinan la presencia de la misma en pacientes previa a la exploración, sugiriendo que el mismo proceso de la enfermedad subyacente predispondría a desarrollar una estenosis uretral. Durante el seguimiento que llevamos a cabo en nuestra serie no objetivamos ninguna estenosis uretral.

Respecto a la actitud terapéutica a adoptar, dada la escasa respuesta de los tratamientos tradicionales utilizando analgésicos, anticolinérgicos y antibióticos que recoge la literatura médica, y basándonos en nuestra experiencia, donde 9 $(75 \%)$ de los pacientes responden a un cambio del hábito miccional en un periodo de 12 meses, esta sería la única medida adoptar en estos casos. En el estudio de Herz ${ }^{13}$, obtiene unas tasas de curación elevadas (83\%) por lo que al igual que el autor proponemos que la uretrorragia en el niño no debe ser considerada idiopática sino consecuencia de una disfunción de vaciado y debe ser tratado como tal.

\section{CONCLUSIÓN}

La uretrorragia en el niño es un proceso benigno en la gran mayoría de los casos que se resuelve de forma espontánea prácticamente siempre durante los 2 primeros años de seguimiento. Los estudios radiológicos y las exploraciones endoscópicas resultan innecesarias en el manejo inicial de estos pacientes quedando relegadas a cuadros persistentes en el tiempo. Del mismo modo, debemos empezar a considerarla dentro de un síndrome que engloba otras manifestaciones consecuencia de un déficit de vaciado y tratarla como tal, intentado evitar exploraciones cruentas y actitudes terapéuticas innecesarias.

\section{REFERENCIAS}

1. Kaplan GW, Brock WA. Idiopathic urethrorrhagia in boys. J Urol. 1982;128(5):1001-1003.

2. Harrison SC, Whitaker RH. Idiopathic urethritis in male children. Br J Urol. 1987;59(3):258-260.

3. Dewan PA, Wilson TM. Idiopathic urethritis in the adolescent male. Eur Urol. 1996;30(4):494-497.

4. Docimo SG, Silver RI, Gonzalez R, Muller SC, Jeffs RD. Idiopathic anterior urethritis in prepubertal and pubertal boys: pathology and clues to etiology. Urology 1998;51(1): 99-102.

5. Datuashvili TD. Profuse urethrorrhagia as a complication of gonorrheal cavernitis. Vestn Dermatol Venerol 1988; 10: 62-63.

6. Kaplan GW, Brock WA. Urethral strictures in children. J Urol. 1983;129(6):1200-1203.

7. Segal AS, Kan Ia D, Dunaevskii Ia L, Dolgopiatov DG. Benign neoplasms of the urethra in men. Urol Nefrol. (Mosk) 1996;(3):39-42.

8. Mazeman E, Bailleul JP, Biserte J. Fibrous polyp of the posterior urethra. 3 cases (author's transl). J Urol. (Paris) 1980;86(8):577-582.

9. Cendron J, Melin Y, Baviera DE, Alaoui-Drai A. Polyp of the posterior urethra. Apropos of 6 cases. Chir Pediatr. 1985;26(6):356-361.

10. Van Howe RS, Cold CJ. Etiology of idiopathic anterior urethritis. Urology 1999;53(3):658.

11. Walker BR, Ellison ED, Snow BW, Cartwright PC. The natural history of idiopathic urethrorrhagia in boys. J Urol. 2001;166(1):231-232.

12. Cuttica RJ, Scheines EJ, Garay SM, Romanelli MC, Maldonado Cocco JA. Juvenile onset Reiter's syndrome. A retrospective study of 26 patients. Clin Exp Rheumatol. $1992 ; 10(3): 285-288$.

13. Herz D, Weiser A, Collette T, Reda E, Levitt S, Franco I. Dysfunctional elimination syndrome as an etiology of idiopathic urethritis in childhood. J Urol. 2005;173(6):2132-2137.

14. Williams DI, Mikhael BR. Urethritis in male children. Proc R Soc Med. 1971;64(2):133-134.

Correspondencia autor: Dr. C. Domínguez Hinarejos.

Servicio de Urología. Hospital Infantil La Fe. Avda. Campanar, 21. 46009 Valencia. Tel.: 963862700

E-mail autor: dominguezhinar@hotmail.com

Información artículo: Original - Urología pediátrica

(Trabajo aceptado septiembre 2006) 